\title{
Null Quaternionic Curves in Semi-Euclidean 3-Space of Index $\nu$
}

\author{
A. Tuna AKsOY*, A.C. ÇÖKen \\ Süleyman Demirel University, Department of Mathematics, Isparta, Turkey \\ Akdeniz University, Department of Mathematics, Antalya, Turkey
}

\begin{abstract}
In this study, we construct the Cartan frame of a null quaternionic curves in semi-Euclidean spaces $\mathbb{R}_{1}^{3}$ and $\mathbb{R}_{2}^{3}$ for an arbitrary parameter.

DOI: 10.12693/APhysPolA.128.B-286

PACS: $02.40 . \mathrm{k}, 02.40 . \mathrm{Hw}$
\end{abstract}

\section{Introduction}

The quaternions were first described by Irish mathematician Sir William Rowan Hamilton in 1843. The space of quaternions $Q$ are isomorphic to $E^{4}, 4$ dimensional vector space over the real numbers. The set of the quaternions, which was introduced by Hamilton, can be represented as $Q=\left\{a e_{1}+b e_{2}+c e_{3}+d\right.$; $a, b, c, d \in \mathbb{R}\}$. Here, $e_{i}^{2}=-1, e_{i} \times e_{j}=e_{k}$, $1 \leq i \leq 3$ and $(i j k)$ is an even permutation of (123). There are a lot of papers associated with quaternions. Also they are used in both theoretical and applied mathematics. Hence, the importance of the study of quaternions and its presence in the physical theories are clear.

In 1987, the Serret-Frenet formulae for quaternionic curves in $E^{3}$ and $E^{4}$ were given by Bharathi and Nagaraj [1]. After that, split quaternions are identified with semi-Euclidean space $E_{2}^{4}$, while the vector part of split quaternions are identified with Minkowski 3-space by Inoguchi [2]. Many studies have been published on the quaternionic curves using this results. Among them Tuna has defined the Serret-Frenet formulae for a quaternionic curve in the semi-Euclidean space $E_{2}^{4}[3]$. We have studied quaternion valued functions and quaternionic inclined curves in the semi-Euclidean space $E_{2}^{4}[4]$. But, to our knowledge, there has been no study on the SerretFrenet formulae for null quaternionic curves in the semiEuclidean spaces. In this paper, we will study SerretFrenet formulae for null quaternionic curve in $\mathbb{R}_{1}^{3}$ and null quaternionic curve in $\mathbb{R}_{2}^{3}$.

It is well known that there exist spacelike quaternionic curve and timelike quaternionic curve in the semiEuclidean spaces. However, null quaternionic curves have many properties which are very different from spacelike quaternionic and timelike quaternionic curves. In geometry of null quaternionic curves difficulties arise because the arc length vanishes, so that it is impossible to normalize the tangent vector in the usual way. Since the length

*corresponding author; e-mail: abidebytr@yahoo.com of any null curve is zero, we use an affine paramerter, called a distinguished paramerter which plays the role similar to the role of an arc-length paramerter for nonnull curve. The rules of calculus are the same as in Euclidean space.

However, since any $\mathbb{R}_{\nu}^{3}$ can have a null quaternionic curve (for example, any null vector can generate a null curve) as stated before the calculus of null quaternionic curve is quite different than the non-null curve. On the other hand, since each null vector has zero length, the usual arc length parametrization is not possible for a null curve. The smooth curve $\mathcal{C}$ is said to be a null curve if the tangent vector to $\mathcal{C}$ at any point is a null vector $[5,6]$.

Bonnor introduces the Cartan frame as the most useful one and he uses this frame to study the behaviors of a null curve [7]. Thus, one can use these fundamental results as the basic tools in researching the geometry of null curves.

Thus, the current study hopes to serve such a need; in this paper, we study the Serret-Frenet formulae and the associated with a null quaternionic Cartan curve in semiEuclidean 3-space with index $\nu=\{1,2\}$.

\section{Preliminaries}

Let $\mathcal{C}$ be a smooth curve immersed in an $(m+2)$ dimensional proper semi-Riemannian manifold $\left(M_{q}^{m+2} g\right)$ of a constant index $q \geq 1$. Then, there exists a quashiorthonormal basis along a null curve $\mathcal{C}$ of a proper semiRiemannian manifold $\left(M_{q}^{m+2} g\right)$. We show, here, how to construct some special basis of semi semi-Euclidean spaces along a lightlike subspace.

Let $(V, g)$ be an $m$-dimensional proper semi-Euclidean space whose quadratic form is of type $(p, q, 0), p+q=$ $m$ and $p \cdot q \neq 0$. Consider an orthonormal basis $\left\{e_{1}, e_{2}, \ldots e_{m}\right\}$ of $V$ such that $\left\{e_{1}, e_{2}, \ldots e_{q}\right\}$ and $\left\{e_{q+1}, \ldots e_{q+p}\right\}$ are unit timelike and spacelike vectors, respectively. To construct a basis including some lightlike vectors we analyse the following cases.

Case I: $(q<p)$. Let us construct vectors

$$
f_{i}=\frac{1}{\sqrt{2}}\left(e_{q+i}+e_{i}\right) ; f_{i}^{*}=\frac{1}{\sqrt{2}}\left(e_{q+i}-e_{i}\right), i \in 1,2, \ldots q
$$

which satisfy $g\left(f_{i}, f_{j}\right)=g\left(f_{i}^{*} f_{j}^{*}\right)=0$ and $g\left(f_{i}, f_{j}^{*}\right)=$ 
$\delta_{i j} i j \in 1,2, \ldots q . \quad$ Thus $\left\{f_{1}, \ldots f_{q}, f_{1}^{*}, \ldots f_{q}^{*}\right.$, $\left.e_{2 q+1}, \ldots e_{q+p}\right\}$ is a basis of $\mathcal{V}$ which contains $2 q$ lightlike vectors and $p-q$ spacelike vectors.

Case II: $(p<q)$ The basis $\left\{f_{1}, \ldots f_{p}, f_{1}^{*}, \ldots f_{p}^{*}\right.$, $\left.e_{p+1}, \ldots e_{q}\right\}$ contains $2 p$ lightlike vectors and $q-p$ timelike vectors.

Case III: $(p=q)$. Since $m=2 q=2 p$ we obtain the lightlike basis $\left\{f_{1}, \ldots f_{q}, f_{1}^{*}, \ldots f_{q}^{*}\right\}[5]$.

Let we give basic concept about the semi-real quaternions. The set of the semi real quaternions

$$
\begin{gathered}
Q_{\nu}=\left\{q \mid q=a e_{1}+b e_{2}+c e_{3}+d ; a, b, c, d \in \mathbb{R},\right. \\
e_{1}, e_{2}, e_{3} \in \mathbb{R}_{\nu}^{3}(\nu=1,2), \\
\left.h_{\nu}\left(e_{i}, e_{i}\right)=\varepsilon\left(e_{i}\right), 1 \leq i \leq 3\right\},
\end{gathered}
$$

where

$$
\begin{aligned}
& e_{i} \times e_{i}=-\varepsilon\left(e_{i}\right), 1 \leq i \leq 3 \\
& e_{i} \times e_{j}=\varepsilon\left(e_{i}\right) \varepsilon\left(e_{j}\right) e_{k} \in \mathbb{R}_{1}^{3} \\
& e_{i} \times e_{j}=-\varepsilon\left(e_{i}\right) \varepsilon\left(e_{j}\right) e_{k} \in \mathbb{R}_{2}^{4}
\end{aligned}
$$

and $(i j k)$ is an even permutation of (123). The multiplication of two semi real quaternions $p$ and $q$ is defined by

$$
p \times q=S_{p} S_{q}+S_{p} V_{q}+S_{q} V_{p}+h\left(V_{p}, V_{q}\right)+V_{p} \wedge V_{q}
$$

for every $p, q \in \mathbb{Q}_{\nu}$ where we have used the inner and cross products in semi-Euclidean space $\mathbb{R}_{\nu}^{3}$. For a semi real quaternion $q=a e_{1}+b e_{2}+c e_{3}+d \in \mathbb{Q}_{\nu}$ the conjugate $\alpha q$ of $q$ is defined by $\alpha q=-a e_{1}-b e_{2}-c e_{3}+d$ Thus, we define symmetric, non-degenerate valued bilinear form $h$ as follows:

$$
h_{\nu}: \mathbb{Q}_{\nu} \times \mathbb{Q}_{\nu} \rightarrow \mathbb{R}
$$

by

$$
\begin{aligned}
& h_{1}(p, q)=\frac{1}{2}[\varepsilon(p) \varepsilon(\alpha q)(p \times \alpha q)+\varepsilon(q) \varepsilon(\alpha p)(q \times \alpha p)] \\
& \quad \text { for } \mathbb{R}_{1}^{3}
\end{aligned}
$$

and

$$
h_{2}(p, q)=\frac{1}{2}[-\varepsilon(p) \varepsilon(\alpha q)(p \times \alpha q)-\varepsilon(q) \varepsilon(\alpha p)(q \times \alpha p)]
$$
for $\mathbb{R}_{2}^{4}$,

where

$$
h_{\nu}(q, q)=a^{2} \varepsilon\left(e_{1}\right)+b^{2} \varepsilon\left(e_{2}\right)+c^{2} \varepsilon\left(e_{3}\right)+d^{2}, \nu=\{1,2\}
$$

and it is called the semi-real quaternion inner product. The vector product of two semi real quaternions $p=a_{1} e_{1}+b_{1} e_{2}+c_{1} e_{3}+d_{1}$ and $q=a e_{1}+b e_{2}+c e_{3}+d$ is defined as

$$
\begin{aligned}
& V_{p} \wedge V_{q}=\varepsilon\left(e_{2}\right) \varepsilon\left(e_{3}\right)\left(b_{1} c-b c_{1}\right) e_{1} \\
& -\varepsilon\left(e_{1}\right) \varepsilon\left(e_{3}\right)\left(a_{1} c-a c 1\right) e_{2}+\varepsilon\left(e_{1}\right) \varepsilon\left(e_{2}\right)\left(a_{1} b-a b_{1}\right) e_{3}, \\
& \quad \nu=1
\end{aligned}
$$

and

$$
\begin{aligned}
& V_{p} \wedge V_{q}=-\varepsilon\left(e_{2}\right) \varepsilon\left(e_{3}\right)\left(b_{1} c-b c_{1}\right) e_{1} \\
& +\varepsilon\left(e_{1}\right) \varepsilon\left(e_{3}\right)\left(a_{1} c-a c_{1}\right) e_{2}-\varepsilon\left(e_{1}\right) \varepsilon\left(e_{2}\right)\left(a_{1} b-a b_{1}\right) e_{3}, \\
& \quad \nu=2 .
\end{aligned}
$$

Then, the norm of semi real quaternion $q$ is denoted by

$$
\begin{aligned}
\|q\|^{2} & =\left|h_{\nu}(q q)\right|=\left|a^{2} \varepsilon\left(e_{1}\right)+b^{2} \varepsilon\left(e_{2}\right)+c^{2} \varepsilon\left(e_{3}\right)+d^{2}\right|, \\
\nu & =\{1,2\} .
\end{aligned}
$$

The concept of a spatial quaternion will be made use throughout our work. $q$ is called a spatial quaternion whenever $q+\alpha q=0$. It is a temporal quaternion whenever $q-\alpha q=0[3,4,8]$.

In this paper, we will study Serret- Frenet formulae for null quaternionic curve in $\mathbb{R}_{1}^{3}$ and null quaternionic curve in $\mathbb{R}_{2}^{3}$

Let $\gamma(\mathrm{t})$ be a null quaternionic curve in the 3dimensional semi-Euclidean space $\mathbb{R}_{\nu}^{3}$ of index $\nu$, that is $h\left(\gamma^{\prime}(t), \gamma^{\prime}(t)\right)=0, \gamma^{\prime}(t) \neq 0$. If $h\left(\gamma^{\prime \prime}(t), \gamma^{\prime \prime}(t)\right) \neq 0$ then we may consider the pseudo arc paramerter and Frenet equation with Cartan curvature.

Now let us begin to set up the Frenet equations of a null quaternionic curve by using the main results of (see [5] for null curves)

\section{Serret-Frenet formulae for null quaternionic curves in the semi-Euclidean spaces $\mathbb{R}_{1}^{3}$}

Case I: (Let $e_{1}$ be a timelike vector.)

Let $\left\{e_{1}, e_{2}, e_{3}\right\}$ of $\mathbb{R}_{1}^{3}$. Let $e_{1}$ be a timelike vector. Constract vectors

$$
l=\frac{1}{\sqrt{2}}\left(e_{2}+e_{1}\right), n=l=\frac{1}{\sqrt{2}}\left(e_{2}-e_{1}\right), u=e_{3}
$$

which satisfy $h(l, l)=h(n, n)=h(l, u)=h(n, u)=0$ and $h(l, n)=h(u, u)=1$. Thus $\{l, n, u\}$ is a basis of $\mathbb{R}_{1}^{3}$ which constains $l$ and $n$ null vectors and $u$ spacelike vector. Here, the quaternion product $\times$ given by

$$
\begin{aligned}
& l \times n=-1-u, n \times l=-1+u, n \times u=-n, \\
& u \times n=n, u \times l=-l, l \times u=l, u \times u=-1, \\
& l \times l=0, n \times n=0
\end{aligned}
$$

The three-dimensional semi-Euclidean space $\mathbb{R}_{1}^{3}$ is identified with the space of null spatial quaternions $\left\{\gamma \in \mathbb{Q}_{\mathbb{R}_{1}^{3}} \mid \gamma+\alpha \gamma=0\right\}$ in an obvious manner.

$\gamma: I \subset \mathbb{R} \rightarrow \mathbb{Q}_{\mathbb{R}_{1}^{3}}, s \rightarrow \gamma(s)=\sum_{i=1}^{3} \gamma_{i}(s) e_{i}$,

$1 \leq i \leq 3$. Let $\{l, n, u\}$ be the Frenet trihedron of the differentiable semi-Euclidean space null spatial quaternionic curve in the semi-Euclidean space $\mathbb{R}_{1}^{3}$.

The Frenet equations are

$$
l^{\prime}=k u, n^{\prime}=\tau u, u^{\prime}=-\tau l-k n
$$

and

$$
\begin{aligned}
& l^{\prime}=k u, n^{\prime}=-\tau u, \\
& u^{\prime}=\tau l-k n,
\end{aligned}
$$

where $k$ and $\tau$ are the curvature and torsion.

Let $\mathbb{R}_{1}^{3}$ denote a 3 -dimensional semi-Euclidean space with quaternionic metric $h$. A curve $\gamma(s)$ in $\mathbb{R}_{1}^{3}$ is a null quaternionic curve if $h\left(\gamma^{\prime}(s), \gamma^{\prime}(s)\right)=0$ and $\gamma^{\prime}(s) \neq 0$ for all $s$. We note that a null quaternionic curve $\gamma(s)$ in $\mathbb{R}_{1}^{3}$ 
satisfies $h\left(\gamma^{\prime \prime}(s), \gamma^{\prime \prime}(s)\right) \neq 0$. We say that a null quaternionic curve $\gamma(s)$ in $\mathbb{R}_{1}^{3}$ is parametrized by the pseudo-arc if $h\left(\gamma^{\prime \prime}(s), \gamma^{\prime \prime}(s)\right)=1$. Let $\gamma(s)=\gamma_{1}(s) e_{1}+\gamma_{2}(s) e_{2}+$ $\gamma_{3}(s) e_{3}$ be a null quaternionic curve in $\mathbb{R}_{1}^{3}$. The tangent vector $l=\gamma^{\prime}(s)$ and

$$
h(l, l)=l \times \alpha l=0
$$

has zero length. Differentiating of this equation with respect to $s$,

$$
l^{\prime} \times \alpha l+l \times \alpha l^{\prime}=0
$$

is found. From Eq. (3.2),

- $h\left(l^{\prime}, l\right)=0$,

- $l^{\prime} \times \alpha l$ is a null spatial quaternion

is obtained. Since $\gamma$ is a null, we have $h\left(\gamma^{\prime \prime}, \gamma^{\prime}\right)=0$. But we don't allow $\gamma^{\prime \prime}=u$ to be null, so it is always spacelike. We also have $h\left(\gamma^{\prime \prime}, \gamma^{\prime \prime}\right)+h\left(\gamma^{\prime}, \gamma^{\prime \prime \prime}\right)=0$ then $h(u, u)+h\left(l, u^{\prime}\right)=0$ can be written. Here, $h\left(l, u^{\prime}\right)=-1$ is written. Since $l^{\prime}$ itself is a spatial quaternion, we define the spatial quaternion $u$ and the scalar function $k$ by means of the following equation

$$
l^{\prime}=k u, k=\left\|l^{\prime}\right\| .
$$

Hence, substituting Eq. (3.3) in the Eq. (3.2) we obtain that $h(l, u)=0$, where $l$ is orthogonal to $u$. Now, $l^{\prime}$ is a vector and can be written in terms of any basis. With the orthonormal basis $\{l, n, u\}, l^{\prime}$ becomes,

$$
l^{\prime}=a l+b n+c u, a, b, c \in \mathbb{R} .
$$

Taking the scalar product of equation (3.4) with $l, n$ and $u$, respectively. $a=0, b=0$ and $c=k$ is obtained. We find that $l^{\prime}=k u$. It is easy to see that $l^{\prime}=\gamma^{\prime \prime}(s)$ and then $u(s)=\frac{\gamma^{\prime \prime}(s)}{\left\|\gamma^{\prime \prime}(s)\right\|}$, so that we write

$$
l^{\prime}(s)=\left\|l^{\prime}(s)\right\| u(s) .
$$

Taking the scalar product of Eq. (3.5) with $u(s)$. Thus, by using the equation $h\left(l^{\prime}, u\right)=k$, we obtain $k=\left\|l^{\prime}(s)\right\|$. On the other hand, differentiating this equation $l \times n=-1-u$ and then we arrange $l \times n^{\prime}=-u^{\prime}-k n$ is obtained. Here, $-u^{\prime}-k n$ is orthogonal to $l$ and $u,\left(-u^{\prime}-k n\right)$ is parallel to $l \times u$ and $u \times l$.

$$
u^{\prime}=-\tau l-k n \text { and } u^{\prime}=\tau l-k n
$$

are found. Similarly, differentiating this equation $n \times l=-1+u$ and doing the necessary arrengements, $u^{\prime}+k n$ is parallel to $l \times u$ and $u \times l$

$$
u^{\prime}=-\tau l-k n, u^{\prime}=\tau l-k n
$$

are obtained. The last equation has the same as the equation (3.6)

$$
n^{\prime}=a l+b n+c u, a, b, c \in \mathbb{R} .
$$

Taking the scalar product of Eq. (3.8) with $l$ and $n$, respectively,

$$
a=0 \text { and } b=0
$$

are obtained. Again in the same way, if it is done with inner product $n^{\prime}$ vector by $u$ vector, we get $h\left(n^{\prime}, u\right)=c$. When we differantiate the equation $h(n, u)=0$, we get $h\left(n^{\prime}, u\right)+h\left(n, u^{\prime}\right)=0$. And when we consider that the vector $u^{\prime}$ has two different values here; we get $n^{\prime}=\tau u$ and $n^{\prime}=-\tau u$. Thus, we may express Frenet formulae of the Frenet trihedron in the matrix form:

$$
\left[\begin{array}{l}
l^{\prime} \\
n^{\prime} \\
u^{\prime}
\end{array}\right]=\left[\begin{array}{ccc}
0 & 0 & k \\
0 & 0 & \tau \\
-\tau & -k & 0
\end{array}\right]\left[\begin{array}{l}
l \\
n \\
u
\end{array}\right]
$$

and

$$
\left[\begin{array}{l}
l^{\prime} \\
n^{\prime} \\
u^{\prime}
\end{array}\right]=\left[\begin{array}{ccc}
0 & 0 & k \\
0 & 0 & -\tau \\
\tau & -k & 0
\end{array}\right]\left[\begin{array}{l}
l \\
n \\
u
\end{array}\right]
$$

Case II: (Let $e_{2}$ be a timelike vector.)

Let $\mathbb{R}_{1}^{3}$ denote a 3-dimensional semi-Euclidean space with quaternionic metric $h$. Let $\gamma: I \rightarrow \mathbb{R}_{1}^{3}$ be a null quaternionic Cartan curve such that $\left\{\gamma^{\prime}(s), \gamma^{\prime \prime}(s), \gamma^{\prime \prime \prime}(s)\right\}$ is positively or negatively oriented for all $s \in I$. Let us consider its Cartan frame $\left\{l=\gamma^{\prime}(s), n, u\right\}$ where $h(l, l)=h(n, n)=h(l, u)=h(n, u)=0, h(l, n)=$ $-1, h(u, u)=1$ with the quaternion product $\times$ given by $l \times l=n \times n=0, u \times u=-1, l \times n=1-u, n \times l=$ $1+u,-(u \times n)=n=n \times u$ and $-(l \times u)=l=u \times=l$. The Frenet equations are

$$
l^{\prime}=k u, n^{\prime}=-\tau u, u^{\prime}=-\tau l+k n,
$$

and

$$
l^{\prime}=k u, n^{\prime}=\tau u, u^{\prime}=\tau l+k n,
$$

where $\tau$ denotes covariant derivative and $\mathrm{k}$ is the curvature of the curve. Then, we can do as proof of Case I.

\section{Serret-Frenet formulae for null quaternionic curves in the semi-Euclidean spaces $\mathcal{R}_{2}^{3}$}

Case I: (Let $e_{1}$ and $e_{2}$ be two timelike vectors.)

Let $\left\{e_{1}, e_{2}, e_{3}\right\}$ be an ortonormal basis of $\mathbb{R}_{2}^{3}$ and $e_{1}$ and $e_{2}$ two timelike vectors. Constract vectors

$$
l=\frac{1}{\sqrt{2}}\left(e_{3}+e_{1}\right), n=\frac{1}{\sqrt{2}}\left(e_{3}-e_{1}\right), u=e_{2}
$$

which satisfy $h(l, l)=h(n, n)=h(l, u)=h(n, u)=0$, $h(l, n)=1, h(u, u)=-1$. Thus $\{l, n, u\}$ is a basis of $\mathbb{R}_{2}^{3}$ which constains $l$ and $n$ null vectors and $u$ timelike vector. Here, the quaternion product $\times$ given by

$$
\begin{aligned}
& l \times n=-1-u, n \times l=-1+u, \\
& n \times u=n=-(u \times n), u \times l=l=-(l \times u)=l, \\
& u \times u=1, l \times l=0, n \times n=0 .
\end{aligned}
$$

The Frenet equations are

$$
l^{\prime}=k u, n^{\prime}=-\tau u, u^{\prime}=-\tau l+k n,
$$

and

$$
l^{\prime}=k u, n^{\prime}=\tau u, u^{\prime}=\tau l+k n,
$$

where $k$ and $\tau$ are the curvature and torsion.

The three-dimensional semi-Euclidean space $\mathbb{R}_{2}^{3}$ is identified with the space of null spatial quaternions $\{\gamma \in$ $\left.\mathbb{Q}_{\mathbb{R}_{2}^{3}} \mid \gamma+\alpha \gamma=0\right\}$ in an obvious manner. $\gamma: I \subset$ $\mathbb{R} \rightarrow \mathbb{Q}_{\mathbb{R}_{2}^{3}}, s \rightarrow \gamma(s)=\sum_{i=1}^{3} \gamma_{i}(s) e_{i}, 1 \leq i \leq 3$. Let 
$\{l, n, u\}$ be the Frenet trihedron of the differentiable semiEuclidean space null spatial quaternionic curve in the semi-Euclidean space $\mathbb{R}_{2}^{3}$.

Let $\mathbb{R}_{2}^{3}$ denote a 3 -dimensional semi-Euclidean space with quaternionic metric $h$. A curve $\gamma(s)$ in $\mathbb{R}_{1}^{3}$ is a null quaternionic curve if $h\left(\gamma^{\prime}(s), \gamma^{\prime}(s)\right)=0$ and $\gamma^{\prime}(s) \neq 0$ for all $s$. We note that a null quaternionic curve $\gamma(s)$ in $\mathbb{R}_{2}^{3}$ satisfies $h\left(\gamma^{\prime \prime}(s), \gamma^{\prime \prime}(s)\right) \neq 0$. We say that a null quaternionic curve $\gamma(s)$ in $\mathbb{R}_{2}^{3}$ is parametrized by the pseudo-arc if $h\left(\gamma^{\prime \prime}(s), \gamma^{\prime \prime}(s)\right)=-1$. Let $\gamma(s)=\gamma_{1}(s) e_{1}+\gamma_{2}(s) e_{2}+$ $\gamma_{3}(s) e_{3}$ be a null quaternionic curve in $\mathbb{R}_{2}^{3}$. The tangent vector $l=\gamma^{\prime}(s)$ and $h(l, l)=l \times \alpha l=0$ has zero length. Since $\gamma^{\prime}$ is a null, we have $h\left(\gamma^{\prime \prime}, \gamma^{\prime}\right)=0$. But we don't allow $\gamma^{\prime \prime}=u$ to be null, so it is always timelike. We also have $h\left(\gamma^{\prime \prime}, \gamma^{\prime \prime}\right)+h\left(\gamma^{\prime}, \gamma^{\prime \prime \prime}\right)=0$ then $h(u, u)+h\left(l, u^{\prime}\right)=0$ can be written. Here, $h\left(l, u^{\prime}\right)=1$ is written.

Then, we can do as proof of Case I.

Case II: (Let $e_{2}$ and $e_{3}$ be two timelike vectors.)

Let $\mathbb{R}_{2}^{3}$ denote a 3 -dimensional semi-Euclidean space with quaternionic metric $h$. Let $\gamma: I \rightarrow \mathbb{R}_{2}^{3}$ be a null quaternionic Cartan curve such that $\left\{\gamma^{\prime}(s), \gamma^{\prime \prime}(s)\right.$, $\left.\gamma^{\prime \prime \prime}(s)\right\}$ is positively or negatively oriented for all $s \in I$. Let us consider its Cartan frame $\left\{l=\gamma^{\prime}(s), n, u\right\}$ where

$$
\begin{aligned}
& h(l, l)=h(n, n)=h(l, u)=h(u, n)=0, \\
& h(l, n)=h(u, u)=-1
\end{aligned}
$$

with the quaternion product $x$ given by

$$
\begin{aligned}
& l \times n=1-u, n \times l=1+u,-(n \times u)=n=u \times n, \\
& l \times u=l=-(u \times l), u \times u=1, l \times l=0, n \times n=0 .
\end{aligned}
$$

The Frenet equations are

$$
l^{\prime}=k u, n^{\prime}=\tau u, u^{\prime}=-\tau l-k n,
$$

and

$$
l^{\prime}=k u, n^{\prime}=-\tau u, u^{\prime}=\tau l-k n,
$$

where $\tau$ denotes covariant derivative and $k$ is the curvature of the curve.

Then, we can do as proof of Case I.

\section{Conclusions}

In case $\gamma$ is a null quaternionic Cartan curve in $\mathbb{R}_{1}^{3}$, while $e_{1}$ be a timelike vector, labeling $k(s)=1$, the Frenet formula of $\gamma(s)$ with respect to $\{l, n, u\}$ becomes

$$
l^{\prime}=u, n^{\prime}=\tau u, u^{\prime}=-\tau l-n,
$$

and

$$
l^{\prime}=u, n^{\prime}=-\tau u, u^{\prime}=\tau l-n .
$$

In case $\gamma$ is a null quaternionic Cartan curve in $\mathbb{R}_{1}^{3}$, while $e_{2}$ be a timelike vector, labeling $k(s)=1$, the Frenet formula of $\gamma(s)$ with respect to $\{l, n, u\}$ becomes

$$
l^{\prime}=u, n^{\prime}=-\tau u, u^{\prime}=-\tau l+n,
$$

and

$$
l^{\prime}=u n^{\prime}=\tau u, u^{\prime}=\tau l+n .
$$

In case $\gamma$ is a null quaternionic Cartan curve in $\mathbb{R}_{2}^{3}$, while $e_{1}$ and $e_{2}$ be two timelike vectors, labeling $k(s)=1$, the Frenet formula of $\gamma(s)$ with respect to $\{l, n, u\}$ becomes

$$
l^{\prime}=u, n^{\prime}=-\tau u, u^{\prime}=-\tau l+n,
$$

and

$$
l^{\prime}=u, n^{\prime}=\tau u, u^{\prime}=\tau l+n .
$$

In case $\gamma$ is a null quaternionic Cartan curve in $\mathbb{R}_{2}^{3}$, while $e_{2}$ and $e_{3}$ be two timelike vectors, labeling $k(s)=1$, the Frenet formula of $\gamma(s)$ with respect to $\{l, n, u\}$ becomes

$$
l^{\prime}=u, n^{\prime}=\tau u, u^{\prime}=-\tau l-n,
$$

and

$$
l^{\prime}=u, n^{\prime}=-\tau u, u^{\prime}=\tau l-n .
$$

\section{Acknowledgments}

This work was supported by the SDU Funding Agency under Grant 2059-D-09.

\section{References}

[1] K. Bharathi, M. Nagaraj, Indian J. Pure Ap. Mat. 18, 507(1987).

[2] J. Inoguchi, Tokyo J. Math. 21, 141 (1998).

[3] A. Tuna, M.Sc. Thesis, Süleyman Demirel University Graduate School of Naturel and Applied Science, Isparta, Turkey 2002.

[4] A.C. Çöken, A. Tuna, Appl. Math. Comput. 155 , 373 (2004).

[5] L.K. Duggal, A. Bejancu, Lightlike submanifolds of semi-riemannian manifolds and applications, Kluwer Academics Publishers, Dordrecht 1996, p. 300.

[6] B.O. Neill, Semi-Riemannian geometry with applications to relativity, Academic Press, London 1983.

[7] W.B. Bonnor, Tensor N.S. 20, 229(1969).

[8] A. Tuna Aksoy, Ph.D. Thesis, Süleyman Demirel University Graduate School of Natural and Applied Science, Isparta, Turkey 2014. 